Aus dem Genesungsheim Hohenwiese der LVA. Schlesien. (Chefarzt: Dr. Hasse.)

\title{
Ueber Spätrachitis.
}

\author{
Von Dr. K. Stetter, Oberarzt.
}

Als Wirkung der Blockade auf die Bevölkerung der Mittelmächte müssen neben der allgemeinen Untercrnährung, der anstcigenden Sterblichkeitsziffer, der crschreckenden Tuberkulosczunahme und dem Hungerödem auch die im letzten Jahre gehäuft auftretenden abnormen Knochenveränderungen erwahnt werden. Nicht allein bei Kindern wurde in letzter Zeit starke Zunalıme von Rachitis beobachtet, und zwar sowohl in der bisher bekannten Erschcinungsform als auch in der Wcise, daß Kinder, die bereits laufen gelernt hatten, allmählich nicht mehr laufen wollen, und da $B$ die Rachitis über das 2 . Lebens. jahr fortdauert, wie $\mathrm{Japha}^{1}$ ) berichitet, sondern es mchren sich scit Anfang dieses Jahres auch die Berichtc über abnorme Knochenerkrankungen bei Erwachsencn. Edelniann ${ }^{2}$ ) und bald darauf Schlesing e ${ }^{3}$ ) und $S c_{\text {iff }}{ }^{4}$ ) berichteten Anfang 1919 aus Wien iiber Knochicnveränderungen bei älteren Leuten im Sinne von Osteomalazie; Schlesinger sprach direkt von Hungermalazie. In Deutschland berichtete als Erster Fromm $\mathrm{C}^{5}$ ) 6) i) yor der Mcdizinischen Gescllschaft Göttingen über Knochıcnveränderungen bei Adoleszenten, dic cr als Spätrachitis mit Uebergang in die Ostcomalazie bezeichncte und von der er in einem zweiten Vortrage im Mai v. Js. bereits über 66 sclbstbeobachtete und 266 von Aerzten der Göttinger Gegend festgestellte Fälle referierte. Dann folgten ähnliche Berichte von Hochstetter ${ }^{8}$ ) aus der Tübinger Klinik über 4 Fälle von Spätrachitis bei Heranwachsenden, von $S$ i mon ${ }^{9}$ ), der bei nuännlichen Adolszenten mehr Rachitisform, bei Mädchen mch Aehnlichkeit mit Ostcomalazie fand, von Böhme ${ }^{10}$ ) aus Bochum uber 20 der Rachitis zugchörige Fälle bei Jugendlichen und 10 osteomalazieähnlichc bei Erwachsencn, von B it to r ${ }^{11}$ ) aus Breslau über Erkrarkungen an Spätrachitis bei 15 - bis 18 jährigen mänılichen Individuen und von Dotze112) über gehäıftes Anftreten von Spätrachitis bei männlichen Adoleszenten in der Bonner Gegend. Partscl113) stcllte in Drcsden einc Hälffung ron osteomalazieähnlichen Zuständeı bei Leıten über 60 Jahren fest, und Alwen sit bcobachtete an 30 Frauen im klimakterischicn und postklimakterischen Alter osteoporotische Erscheinungen.

Es handelt sich also um in Dentsclıland und den übrigen Ländern der blockierten Mittclmächtc zur Bcobachtung kommende, an manchen Orten gehäuft auftrctende Krankheitsbilder, deren gemeinsame Ursachc in der mangclhaften Kriegscrnährung zu suchen ist, während die Erscheinungsformen der Knochenerkrankungen sich deutlich in solche, die das Bild der Spätrachitis bicten (hauptsächlich bci heranwachsenden, meist mánnlichen Individuen), und solche mit dem Charakter der Osteomalazie und Osteoporose (melır bei älteren Leuten) trennen lassen. Dem liicsigen Genesıngsheim für Männcr wurden in der Zeit vom 15. VI. bis 30. X. 191913 Fälle von Spätrachitis überwicsen. Es lıandelte sich um 17- bis 19 jährige Hcranwachsende männlichen Geschlechts, dem Beruf nach meist schwerarbeitende Leute (Schmiede, Schlosser, Tischlcr, Mechanikcr), die während des Krieges schwere Arbeit zum Teil in Nachtschichten verriclitct hatten; nur 2 Kranke gaben an, als Uhirmacher bzw: als Bureaugehilfe leichte Arbeit verrichtet zu haben. Fast alle stammten aus größeren Städten, meist Indıstrieorten, 3 kamen aus ländlichen Bczirken. Der geographischen Lage nach sind Orte in Niederungen nicht häufiger vertreten als höher gclegene Ortc. Hunsichtlich der Hygienc in den Arbeitsstätten wird von allen Kranken angegcben, daß genügend Licht und Luft vorlanden sci, dagegen war der zulr Verfïgung stchende Schlafraum oft lingenügend (nicist 3 bis 4 Personen, einmal 8 Leute in kleincm Sclilafzimuler). Die Verköstigung erfolgte meist in Hauslalte der Eltern, einmal beim Meister nud war kricgsmäßig. Bis auf einen Pflegling, dessen Vater cine kleine Landwirtschaft nit einer Kuh besaß, bekam in den letzten Jahren keiner Milch. Alle bezeiclneten dic Kost als fcttarm; 7 hielten sie der Menge nach fïr ansreichend, dic andercn fïr knapp; 5 gaben an, nurr von den auf Marken zugewicscıcn Lebensmitteln gelebt zu habcn. Im Winter 1916 und 1917 hatten die meisten viel Rüben und später Dörrgemüse konsumiert. ln der Familienanamnesc crgibt sich hinsichtlich der Eltern nichts Besondercs. Von den Geschwistern soll bei einem Kranken cines Jugendrachitis gchabt laaben. Einer erzählt, daß ein 16- und 17 jähriger Bruder die glciche Krankheit, aher in geringerenı Grade liabe, ein andercr sagt das Gleiche ron scincem 16 jährigen Sticfbruder. Dic Untersıchten selbst wollen anßer Kinderkrankheiten nic crnstlich krank gewcsen scin, ciner soll seit der Sclunlzeit blutarm scin. Ueber Jugendrachitis wissen sic nichts Bestimmtes antnigeben, doch sollen 3 crst nach $11 \%$ Jahren lanfen gelernt haben.

Der Beginn der jetzigen Krankheit liegt viermal 3 Jalıre, dreimal 2 Jahre, scchsnial etwa 1 Jall zuriick und sctzte meist mit leichtcr Ermüdbarkcit in den Beinen," mit Schmerzen in den Knien bei Belastung, hauptsächlich morgens nach denl Aufstehen, und mit Schwer-

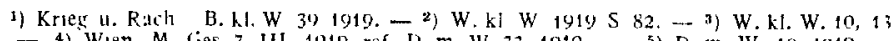
$1919 .-4)$ When, M. Cies. 7 III. 1919 ref. D. m W. 33 1919. 5) D. In. W. 19 1919.

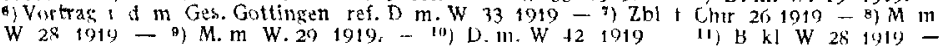
12) Demonst. aus niederrhem. Ges f. Nat. u. Hik, ref D m. W $361919-$ 1.) D. m W. 41
1919. - 11) M. m. W. 38 1919. 
fälligkeit beim Treppensteigen ein. Später kamen Kreuz- und Rückenschmerzen dazu, und in selteneren Fallen kam es zu Verkrünımungen der Beine. Die Beschwerden, welclie besonders im Winter stärker waren und sich auch sonst bei Witterungsweclisel versclilimmerten, zwangen meist zu mehr oder weniger langer Arbeitseinstellung. Bei Aufnahme in das Genesungshein war nur 1 Kranker niclit außer Arbeit gewesen; 4 waren vorher 2 Monate, die ubrigen langer, bis zu $3 / 4$ Jahr krank und arbeitsunfalıig gewesen. Meist war das Leiden längere Zeit, aber erfolglos als Rheumatismus behandelt worden.

Von den im Größenwachstunı fast allgencin etwas zurückgebliebenen Kranken (144 bis $162 \mathrm{~cm}$ groß) waren bei einem Durchsclinittsgewicht von $42 \mathrm{~kg}$ nur 5 mliaig kräftig, $T$ schwaclılich und $1 \mathrm{schr}$ schwächliclı. Blonde Indıvidueı wareı $n$ cht häu iver iertreteı als duukle. 2 wiesen Degenerations zcichen auf (Kryptorclismus, gespaltene Uvula), 1 hatte hypertrophische Tonsillen. Anl den inneren Organten fand sich keine besondere Veränderung. Leber ind Milz waren nie vergrößert, 3 mal bestand leiclıte Sclïlddrüsenhyperplasic, 2 mal fand sich ein mäßig großer Kropfknoten. Nebenuieren*, Thynıısund Hypopliysendysfunktion ließ sich nicht nachweisell. 3 Fälle zeigten leicht gesteigerte Nervenerregbarkeit, einer davon aluch im Fazialisgebiet. Tetanic wurde nicht beobaclitet. 5 mal wurde spontan über gesteigerte Schweißabsonderung geklagt. Katarakt kam nicht zur Beobaclitung. Der Hämoglobingehalt des Blites schwankte zwischen 60 und $70 \%$, die Zahl der roten und weißen Blutzellen war nicht wesentlich verändert, im weißen Blutbilde fiel eine mäßige Vermehrung der Lymphozyten auf Kosten der polynukleären Zellen aüf. Dic äußeren Lymphdrüsen waren niclıt tastbar. In 2 Fällell konnte durch das Vorhandensein stark rachitischer Zähne auf überstandene Jugendrachitis gesclılossen werden.

Was die frischen rachitischen Zeichen anlangt, so war die Epiphysengegend am deutlichsten an den Hand- und Kniegelenken aufgetrieben, nur bei 2 leichteren Fällen fand sich keine siclitbare Verdickung. Rachitischer Rosenkranz konnte bei allen Erkrankten nachrewiesen werden, Deformicrungen an Brustkorb, Wirbelsäule und Becken fehlten. lin Langenwachstum der Knochen traten mcBbare proportionelle Abnormitäten nicht zutage. Der Gang war in über der Hälfte der Fälle melır oder minder watschelnd; Treppenstcigen crfolgte $6 \mathrm{mal}$ langsam und schwerfällig, Stulılsteigen war in diescı Fällen nur mit Unterstützung der oberen Extremitäteı nuögliclı. Bei 3 Kranken mit stark watschelndem Gange konnte leiehte Coxa varaBildung nachgewiesen werden nit einem Trochanterstand bis zu $1,5 \mathrm{~cm}$ über der Roser-Nelatonschen Linic. Bei den übrigen Kranken ließ sich auch röntgenologisch keinc Verbiegung am Schenkelhals féststelleı, dagegen war das Spreizen der Beiıc und das Erhebeı der im Knie gestreckten Bcine leicht behindert, sodaß wolıl auch cine gewisse Muskelschwäche beim Zustandekommen des watschelndell Ganges mitspielt. An den oberen Extremitäten kamen Verkrimmungen der Knochen nicht zur Beobachtung, dagegen zeigten nur 3 Kranke normale Bildung der unteren Extremitäten, die ja am meisten der Belastung ausgesetzt sind. 6 mal bestand Genu varum mit Abstand der inneren Fenıurepikondylen von etwa $3 \mathrm{~cm}, 1 \mathrm{mal}$ von $6 \mathrm{~cm}$ und $1 \mathrm{mal}$ von $9 \mathrm{~cm}$, wobes im letzten Falle bereits doppelseitige Osteotomie der Tibia ausgeführt war. 2 Beobachtete zeıgten X-Beinstellung mit Distanz der inneren FuBknöchel von 9 und $10 \mathrm{~cm}$. Frakturen und Infraktionen kamen nicht zur Beobachtung, ebenso keine Infiltrationsherde in der Muskulatur. Ueber abnorm starke Zahnkaries, die sich erst in den beiden letzten Jahren entwickelte, klagten 3 Kranke.

Charakteristische röntgenologische Befunde wurden bei allen unseren Fällen erhoben. Das hängt wohl damit zusammen, daß nur Kranke mit ausgeprägten Symptomen denı Heime überwiesen

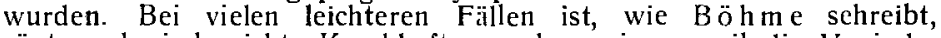
röntgenologisch nichts Krankliaftes nachzuweisen, weil die Veranderungen zu gering sind, um röntgenologische Spuren zu hinterlassen. Wie bei der Jugendrachitis findet man Auftreibung der Epiphysengegend, Verbreiterung der Wachstums zone und Atrophie des Knoclıens. Am besten stellen sich die Veränderungen an den niclıt selır dicken Vorderarmknochen dar. 1)ie Grenzlinie zwischen Epi- und Diapliyse, die bei gesunden Gleichaltrigen meist nur mehr als schnaler, scharf begrenzter Spalt nachzuweisen ist und manchmal wegen bereits eingetretener Verknöcherung ganz fehlt, ist bei den Raclitiskranken durch überschicßende Bilduing ion osteoidem Gewebe verbreitert, 1': coll und darü ver breit. An nıanchen Bilderı ist diese Lücke ganz durchscheillend, all andern zeigt sie gratue, verwaschene Zeichnung, in der derbere Strange (wohl Zeichen von beginnender Verkalkung) nachfuweisen sind. Die den Spalt begrenzenden Teile der Epiphyse und besonders der Diaphyse erscheinen unregelmäßig gezackt, wie ausgefressen, der Diaplyysenteil ist becherartig sertieft und in den Außenkonturen aufgetrieben, wie sich besonders deutlich an der Ulna zeigt. Der der verbreiterten Zone anliegende Teil der Diaphyse ist mianchnial grau verwaschen, scheint massiver und ist oft etwas breiter als normal; in anderen anscheinend leichteren Fallen, wo dic Waclistumszone wenig verbreitert ist, ist dic Ballkchenzeichnung 11 dieser Partic sichtbar, aber deutlich rarefiziert. Aehnliche Epiphysenveränderungen finden sich an den Knochen der Knic- und Fußgelenke. Neben diesen Störungen in der Waclistumszone ist auch das Strukturbıld des ganzen übrigen Knochens mangelhaft, die Bälkchenzeichnung prägt sich schwächer aus, die Kortikalis ersclueint weniger kräftig Nach Eisler' ist sogar diese Knoclientrophic das beweisendste
Merkmal, da eine Verbreiterung des Epiplyysenspaltes oft fehle und auch eine nicht verbreiterte Wachstumszone krank sein könne

Daß die durch die Blockade bedingte schlechte Ernährung das gehäufte Alıftreten der Spätrachitis bedingt, steht außer jedem Zweifel; dagegelı ist die Pathogenese der Kranklicit auch jetzt noch nicht geklärt. Reiner Mangel an Kalksalzen kann nicht allein Ursache der Rachitis sein, das äußerte noch kurz vor dem Kriege D ippelt ${ }^{1}$ ), der auf Grund von Stoffwechisel- und histologisclien Untersuchungen nlaclıwies, daß in den meisten Fällen prinıirer Kalkmangel in der Nahrung nichit bestelie, sondern da 3 das Wesen der Rachitis in einer Störung des Kalkstoffwechsels zu suchen sei und daß die Skelettveränderungen eine Folge dieser Stoffwechselstorung seien. $\mathrm{Zu}$ ähnlichen Schlussen kommt jetzt $\mathrm{Hochsinger}^{8}$ ), wenn er betont, daß die Kalkablagerung nicht deshalb behindert sei, weil nicht geniïgend Kalk in der Nahrung vorhanden sei, sondern deshalb, weil das neugebildete Kuochengewebe nincht imstande sei, sich in normaler Weise mit der in hinreichender Menge dem Körpergewebe zugeführten Kalknenge zu inıpraguieren. Anderseits hebt $\mathrm{Herbst}{ }^{\circ}$ wieder hervor, dali der Kalkmangel eine der Hauptursaclien der Ostcoporose sei; gerade bei Adoleszenten fehlte wälırend des Krieges das kalkreiclıste Mittel, die Milch, und infolge der Kriegsernälırung und bei dem all Nalırungsresten reıchen Kote seien die Kalkverluste relativ groß. Neben diesen direkten Ernälırungsschädigungen des Knoclıeıs kommen wahrsclieinlıclı auch noch Störungen in der Funktion der Drisen mit innerer Sekretion in Frage. So berichtet $\mathrm{K} l o \mathrm{tz}^{4}$ ) über gunstige Beeinflussung der Rachitis durch Hypophysenmedikation, $\mathrm{B}$ os $\mathrm{si}^{3}$ ) über einell durch Adrenalin geheilten Fall von Osteonalazie, walırend 11 ach $\mathrm{Curschmann}^{6}$ ) die Osteomalazie eine pluriglanduläre Erkrankung ist, bei der sowohl Hyper- als Hypofunktion der verschiedenen innersekretorischen Drüsen gefunden werde. Ziviselıen Rachitıs und Ostcomalazie darf man wohl eine weitgehende Achulichkeit, wenn nicht gar Uebereinstimmung in der Entsteliung und im pathologisch-anatomischen Befunde aunehmen, der das eine Mal an einem noch wachsenden Gebilde, das andere Mal an einem fertigen Knochen erloben wird

Unsere Therapie war vor allem darauf bedacht, für ausreichende und abwechslungsreiche Nalırung zu sorgen, in der neben frischem Gemüse auclı die Milch nicht fehlte. Daneben wurden Soolbader, elektrisclıe Lichtbäder, Quarzlichtbestrahlungen, medi',omechanische Behandlung und Massage angewandt. An Arzneimitteln bewährte sich anı besten Phosphorlebertran, der jetzt leider nicht immer in ausreichender Menge zu beschafien ist und zum Teil durcll Caleium phosplioricum ersetzt werden mußte. Suprarenin wurde in einer Dosierung ion 0,3 jeden 3 . Tag bei 2 Kranken ohne Erfolg gegeben: einer der Behandelten zeiote schlıeBliel, leichte nervöse Reizerscheimungen, obwolil anfangs keine Adrenalinmydriasis bestanden liatte. Ebenso hatte Pituglandol in gleicher Dosierung bei einem weiteren Kranken keinen Erfolg.

Naclı durclıschnittliclı 8 wöclıentlicher Behandlung konnten als völlig beschwerdefrei 5 , als gebessert und arbeitsfähig 8 Kranke entlassen werden; letztere waren imstande Spaziergänge ron 3 bis 4 Stunden Dauer ohne Unterbrechung zu machen, waren aber am nächsten Tage nocl sehr matt in den Beinen. Treppensteigen, das bei der Aufnahme am deutlichsten gestört war, ging bei der Entlassung ohne merkliche Behinderung; auch watschelnder Gang fiel nicht niehr auf. Die Knochenverbiegungen hatten in keinem Falle zugenommen, ohne daß von Apparaten Gebrauch gemacht worden war. Die durchıschnittliche Gewichtszunahme betrug $4 \mathrm{~kg}$, der Hämoglobingelialt hob sich um ctwa 10 bis $15 \%$, die Zahl der roten. Blut. Lellen sticg un etwa $1 / 2$ Million.

Um ein weiteres Umsichgreifen der Spätrachitis be: Adoleszenten zu verhuten, empfichlt sich -... wenn irgend nöglich - eine allgemeine ausgiebigere Lebensmittelbelieferung der schwer arbeitenden Heranwachsenden.

\footnotetext{
1) M. m. W. 371919.
} 